\title{
Memory one month after acute carbon monoxide intoxication: a prospective study
}

\author{
D Deschamps, C Géraud, H Julien, F J Baud, S Dally
}

Occup Environ Med 2003;60:212-216

See end of article for authors' affiliations

Correspondence to: Pr S Dally, Department of Internal Medicine and Clinical Toxicology, Fernand Widal Hospital, 75475 Paris Cedex 10: dally@ext.jussieu.fr.

Accepted 20 June 2002
Background: Serious delayed neuropsychological sequelae may complicate carbon monoxide intoxication. The existence of minor manifestations, especially memory disturbances, is not well documented. Aims: To study several memory functions after carbon monoxide intoxication.

Methods: In a prospective study, 32 poisoned patients without risk factors for cognitive disturbances were compared to 32 paired control subjects one month after acute carbon monoxide intoxication (blood carbon monoxide concentration at least $1.0 \mathrm{mmol} / \mathrm{l}$ ), who had been treated with standard conventional therapy. Psychometric tests included Buschke's verbal memory testing, verbal digit span, Corsi's visuospatial span, reaction times, Stroop's colours decoding test, and verbal fluency test.

Results: (1) Memory functions in poisoned subjects were not worse than in the control group and were even better in some areas: learning, word recall, and quality of learning by Buschke's verbal memory testing. Attention was also better in the patients, in whom visual reaction time was shorter than in controls. (2) Results of several memory functions - quality of learning and immediate visual memory-were positively correlated with the initial carbon monoxide level.

Conclusions: In a highly selected subset of patients devoid of risk factors for memory impairment, memory, objectively evaluated by psychometric testing, was not worse one month after carbon monoxide intoxication in patients undergoing standard treatment than in paired control subjects.
A cute carbon monoxide $(\mathrm{CO})$ poisoning is one of the most frequent causes of acute intoxication, ${ }^{1}$ and is the most common cause of household toxic death; it may also result from occupational accidental exposure. In addition to well known risks of immediate morbidity, including cognitive disturbances, ${ }^{2}$ delayed neuropsychological sequelae may occur after a period of improvement or apparent recovery. Many clinical manifestations have been described (seizures, hallucinations, behavioural disorders, extrapyramidal syndrome, cortical blindness, dementia, etc). ${ }^{2}$ In addition to these obvious disorders, more subtle disturbances of cerebral function, particularly of memory, may occur. Experimental data suggest a specific toxicity of $\mathrm{CO}$ on memory functions: in animals, delayed neuronal death occurs in areas involved in memory process. ${ }^{34}$ In humans, subtle cognitive disorders have been reported with a frequency up to $67 \% .^{5}$ However, these neuropsychological sequelae were most often studied without psychometric testing, ${ }^{6-8}$ or the description or the results of the tests were not fully detailed, with no comparison to non-poisoned controls. ${ }^{5}{ }^{10}$ Moreover, confounding factors or potential bias were seldom taken into account.

We performed a prospective study of psychometric testing of memory one month after an acute CO intoxication in 32 patients without other risk factors for cognitive disturbance who had received standard treatment with hyperbaric and/or normobaric oxygen therapy. ${ }^{11}$ The aims were: (1) to investigate differences between patients and paired controls; and (2) to look for a correlation between each parameter and the initial blood CO concentration.

\section{METHODS}

Patients referred for $\mathrm{CO}$ intoxication, whatever the cause, were examined with a view to decide whether or not they should be included in the study. Inclusion criteria were: age between 18 and 60, French maternal language (or perfectly fluent), sufficient educational level to understand and perform the psychometric tests (that is, at least the level normally reached at 10 years, according to a standardised French scale), and concentration of $\mathrm{CO}$ in the first blood sample at least equal to $1.0 \mathrm{mmol} / \mathrm{l}$ (equivalent to $11 \%$ of carboxyhaemoglobin in patients with a normal haemoglobin level). Patients with a disease or a risk factor which might impair memory were not included: excessive alcohol consumption (detected either by a known ingestion above $1 \mathrm{~g} / \mathrm{kg} /$ day or by an increase in serum $\gamma$-glutamyltransferase activity), treatment with a psychotropic drug, psychiatric disease, drug abuse, professional or recreational exposure to solvents or metals, history of central neurological disease (particularly head injury with loss of consciousness, epilepsy), or known infection by HIV virus. Information about symptoms of the intoxication and treatment (normobaric or hyperbaric oxygen therapy) was recorded systematically. Patients freely gave informed consent.

Blood CO concentration was measured by infrared spectroscopy ${ }^{12}$ in a sample taken either at the site of the intoxication or when the patient arrived at hospital. The initial concentration at the end of exposure to the toxic atmosphere was calculated from $\mathrm{CO}$ kinetics, according to the treatment applied before sampling: CO half life was 240 minutes without oxygen treatment and 55 minutes with normobaric oxygen (personal data) which is similar to that reported previously. ${ }^{13}$ Treatment was either normobaric or hyperbaric oxygen therapy, according to normalised criteria, ${ }^{11}$ in addition to supportive therapy as needed. All the included patients were contacted by phone during the week before the examination, to reduce the loss to follow up, and to ascertain the cause, if any.

Each patient was paired with a control with respect to gender, age, and educational level (according to a standardised French scale). The controls were either relatives of the patients, if possible; or if not, relatives of the investigators. The same inclusion criteria were used for the controls and for the patients. The same examiner tested both patients and controls with an open testing procedure. Psychometric tests were performed one month after intoxication. This interval was chosen because the majority of the cases of obvious delayed 


\section{Main message}

- In this prospective comparative and objective study of memory, CO poisoned patients devoid of any risk factor regarding memory impairment did not have worse memories than paired controls.

neurological sequelae occur after a clear period preceding the onset of symptoms between one week and one month. ${ }^{14}$ Tests (table 1) were selected to study several types of memory (long term, short term, and working memories) and different areas of memory (verbal, significant and non-significant, and visuospatial). Other tests addressed disturbances of attention, which is involved in the working of memory. These tests were the verbal Buschke's testing, ${ }^{15}$ verbal digit span, ${ }^{16}$ the visuospatial span of Corsi ${ }_{17}^{17}$ simple reaction time and dual task with simultaneous reaction time and counting, ${ }^{18}$ decoding of the colours of Stroop, ${ }^{19}$ and the verbal fluency test. ${ }^{20}$ Table 2 presents details of the parameters analysed for each test.

Statistical analysis was performed with NCSS software. ${ }^{21}$ The Martinez and Iglewicz test was performed to assess whether the distributions were normal or not. Comparisons were made by means of the paired $t$ test or Wilcoxon's test as appropriate, and Spearman's correlations were performed. Results are expressed as either mean (SEM) or median (range).

\section{RESULTS}

Over four years, 944 consecutive patients were examined in the hospital for suspicion of acute CO intoxication not resulting in death or obvious neurological sequelae; 230 patients had a blood CO concentration in a sample taken on arrival at hospital or at the place of intoxication at least equal to 1.0 $\mathrm{mmol} / \mathrm{l}$; and 192 had at least one non-inclusion criterion (language for 76; age for 55; psychiatric disease, or alcohol or psychotropic drug consumption for 41; other non-inclusion criteria for 20). Thus, only 38 patients were suitable for inclusion, of which 32 were examined ( 20 women, 12 men). CO was the only toxin in 23 and was associated with smoke inhalation in nine. Median age was 27.5 in both patients and controls (range 18-60). Median blood CO concentration in the first blood sample was $2.08 \mathrm{mmol} / \mathrm{l}$ (range 1.00-8.58) which is equivalent to $23 \%$ carboxyhaemoglobin (range 11-96). Median blood CO concentration at the end of exposure, calculated from $\mathrm{CO}$ concentration in the first blood sample, was 2.71 $\mathrm{mmol} / \mathrm{l}$ (range 1.07-8.58) which is equivalent to $30 \%$ carboxyhaemoglobin (range 12-96). The median number of days between intoxication and psychometric testing was 31 (range 26-36). Eight patients experienced a loss of consciousness, 24 were treated with hyperbaric oxygen.

\section{Comparison of patients and controls}

Table 3 presents a comparison of patients and controls. The patients performed better at Buschke's verbal significance trial than controls: the number of patients who remembered all the words at least at the last trial was higher than the number of controls $(28 v 19, \mathrm{p}<0.05)$ and the number of trials needed to learn all 15 words was less $(\mathrm{p}<0.05)$ for patients $(6.7$ (SEM $0.37)$ ) than for controls (8.2 (SEM 0.54)). Recall memory was also better in patients than in controls: 30 minutes after the end of learning, patients remembered more $(\mathrm{p}<0.005)$ words (14.2 (SEM 0.28)) than controls (12.7 (SEM 0.37)). The quality of learning (that is, the efficiency of committing to memory), measured by the list learning test, was better $(p<0.005)$ in patients $(0.75($ SEM 0.02)) than in controls $(0.64$ (SEM 0.03)). On the other hand, working memory and long term memory did not significantly differ between patients and controls.

On the contrary, immediate verbal non-significant memory, assessed with number recall (recall of numbers in the order of recitation), was worse $(\mathrm{p}<0.05)$ in patients $(6.1$ (SEM 0.23)) than in controls (6.7 (SEM 0.21)).

Finally, immediate and non-significant visual memory, evaluated with Corsi's test, did not significantly differ between patients and controls (number of blocks designed 5.3 (SEM $0.15)$ v $5.1(0.15))$.

Attention was better in patients than in controls: simple mean reaction time was lower $(\mathrm{p}<0.05)$ in patients $(270 \mathrm{~ms}$, range 218-747) than in controls (282 $\mathrm{ms}$, range 242-756). Constancy of attention effort was better $(p<0.005)$ in patients: standard deviation was lower: $39 \mathrm{~ms}$ (range 19-356) versus $55 \mathrm{~ms}$ (range 31-1320) in controls. On the contrary, fatigability was higher in patients: the mean reaction time was higher for the second part of the trial than for the first part in patients, and the opposite was observed in controls. The difference between the first and the second part was therefore negative in patients ( $-6.4 \mathrm{~ms}$, range -190 to 49$)$ and positive in controls $(4.7 \mathrm{~ms}$, range -50 to 486$)(\mathrm{p}<0.05)$. Divided attention did not differ between patients and control subjects as results of visual mean reaction time with double task and interference in the colours decoding trial (Stroop's test) were not significantly different.

\section{Relation between initial blood carbon monoxide concentration and memory results}

Results of three memory tests were positively correlated with initial blood CO concentration, calculated from CO concentration in the first blood sample (table 3; fig 1). Learning quality was better when intoxication was greater, as initial CO blood concentration was positively correlated with results of list learning $(r=0.40 ; \mathrm{p}<0.05)$ of Buschke's trial. This was also observed for non-significant, immediate visual memory assessed with Corsi's test $(r=0.42 ; \mathrm{p}<0.05)$. On the contrary, non-significant verbal memory (recall of numbers) was not linked to the initial blood CO concentration. Among tests assessing attention, intra-individual SD of simple reaction time, which investigates constancy of attention effort, was the only test in which results were correlated with initial blood CO concentration: they were better (SD was lower) when initial blood $\mathrm{CO}$ concentration was high $(r=-0.36$; $\mathrm{p}<0.05)$. These three correlations remained significant when excluding the outliers.

Table 1 Functions assessed with the psychometric tests

\begin{tabular}{lll}
\hline Memory & Attention & Divided attention \\
\hline Short term memory & & \\
Non-significant and verbal: digit span & Simple reaction time test & Reaction time test with double task \\
Non-significant and visual: Corsi's test & Verbal fluence test & Colour/word decoding test \\
Working memory and learning & & \\
$\quad \begin{array}{l}\text { Verbal Buschke's test } \\
\text { Long term memory }\end{array}$ & & \\
Verbal Buschke's test & & \\
\hline
\end{tabular}




\begin{tabular}{ll}
\hline \multicolumn{2}{l}{ Table 2 Main functions evaluated by each } \\
parameter & \\
\hline Parameters & Functions \\
\hline Buschke's test & Significant auditory memory \\
Number of trials & Learning \\
Delayed recall & Delayed memory \\
Recognition & Recognition memory \\
Consistent long term retrieval & Long term memory \\
List learning & Quality of learning \\
Short term retrieval & Working memory \\
Digit span (forward and & Short term auditory non-significant \\
backward) & memory \\
Corsi's test & Visuospatial memory \\
Verbal fluency & Attention \\
Visual reaction time & \\
- With simple task & Attention \\
Mean (all presentations) & Reaction speed \\
Standard deviation & Attentional investment constancy \\
Difference between mean & Fatigability \\
reaction times of the 32 first & \\
and the 32 last presentations & \\
- With double task (counting) & Divided attention \\
Variation of mean/mean of & \\
simple reaction & \\
Number of counting errors on & \\
total number of given & \\
numbers & \\
Stroop's colour word test & Divided attention \\
Interference (duration of & \\
decoding - duration of & \\
colour reading) & \\
\hline & \\
\hline &
\end{tabular}

As heterogeneity of age and educational level (two factors that greatly influence results of tests) could have masked correlations between initial blood $\mathrm{CO}$ concentration and results of the tests, investigation for correlations between results of the tests and initial blood CO concentration was performed in the restricted groups of 16 patients with an educational level corresponding to that normally reached at 15 years, and of 26 patients aged less than 40. No significant correlation for other parameters was found (except for mean of simple reaction time in patients with this educational level: $r=0.55$; $\mathrm{p}<0.05$ ).

\section{DISCUSSION}

As a prerequisite to cognitive tests, this study was limited to patients who were very fluent in French and without any risk factors for memory disorder. It focused on delayed neurological effects of CO, but not on immediate neurological symptoms, which are well known in volunteers ${ }^{22}$ and in poisoned patients. ${ }^{23}$ It showed that, one month after CO poisoning, without any obvious sequelae immediately after intoxication, memory of patients receiving standard treatment with normobaric or hyperbaric oxygen was not lower than in paired controls, and was even better for learning and word recall. In patients, attention was also better for several tests, while fatigability was greater. Furthermore, results of several trials of memory and attention were better when initial blood CO level had been high.

Several factors could explain these results. First, inappropriate pairing of patients and controls could have been performed. A plausible reason for better results among controls could be identified in three pairs only: although they had perfect knowledge of the French language, maternal language was not French in two controls; and one patient had taken, before the examination, a $\beta$ blocker drug which could have improved his performance. A new analysis without these three pairs did not change the results: therefore, we do not believe they were caused by inappropriate pairing. Second, in addition to the 32 studied subjects, six patients did not undergo the tests and could have had different results than the examined patients if they had forgotten their appointment because of a memory impairment. All were called by phone before the fixed date and said that they could not come for familial or professional reasons. Therefore, a bias related to loss of follow up cannot explain the results. Third, the study was not conducted with blinded examination, and the behaviour of the psychologist who applied the tests could have

Table 3 Comparison of patients and controls and search for a correlation between initial blood $\mathrm{CO}$ and tests results

\begin{tabular}{|c|c|c|c|c|c|}
\hline \multirow[b]{2}{*}{ Test } & \multicolumn{3}{|c|}{ Comparison: mean (SE) or median (range) } & \multicolumn{2}{|l|}{ Correlation } \\
\hline & Patients & Controls & $\begin{array}{l}\text { Paired } t \text { test or } \\
\text { Wilcoxon }\end{array}$ & \multicolumn{2}{|l|}{$r($ Spearman $)$} \\
\hline \multicolumn{6}{|l|}{ Buschke's test } \\
\hline Number of trials & $6.7(0.37)$ & $8.2(0.54)$ & * & 0.01 & NS \\
\hline Delayed recall & $14.2(0.28)$ & $12.7(0.37)$ & ** & 0.35 & NS \\
\hline Consistent long term retrieval & $61.9(2.73)$ & $64.5(28-100)$ & NS & 0.14 & NS \\
\hline Short term retrieval & $8(3-31)$ & $11.5(3-33)$ & NS & -0.13 & NS \\
\hline List learning & $0.75(0.02)$ & $0.64(0.03)$ & ** & 0.40 & * \\
\hline \multicolumn{6}{|l|}{ Digit span } \\
\hline Forward & $6.1(0.23)$ & $6.7(0.21)$ & * & -0.05 & NS \\
\hline Backward & $4.7(0.24)$ & $4.8(0.24)$ & NS & 0.14 & NS \\
\hline \multicolumn{6}{|l|}{ Corsi's test } \\
\hline Number of blocks & $5.3(0.15)$ & $5.1(0.15)$ & NS & 0.42 & * \\
\hline \multicolumn{6}{|l|}{ Stroop's colour word test } \\
\hline Interference & $58.5(26-163)$ & $55.5(27-140)$ & NS & -0.08 & NS \\
\hline Visual reaction time & & & & & \\
\hline \multicolumn{6}{|l|}{ - With simple task } \\
\hline Mean & $270(218-747)$ & $282(242-756)$ & * & -0.06 & NS \\
\hline Standard deviation & $39(19-356)$ & $55(31-1320)$ & $* *$ & -0.36 & * \\
\hline $\begin{array}{l}\text { Difference between mean reaction times of } \\
\text { the } 32 \text { first and the } 32 \text { last presentations } \\
\text { - With double task (counting) }\end{array}$ & $-6.4(-190$ to 49$)$ & $4.7(-50$ to 486$)$ & * & -0.01 & NS \\
\hline Variation of mean/mean of simple reaction & $0.80(0.04-4.57)$ & $0.94(-206$ to 3.4$)$ & NS & 0.08 & NS \\
\hline $\begin{array}{l}\text { Number of counting errors on total number } \\
\text { of given numbers }\end{array}$ & $0.093(0.017)$ & $0.122(0.17)$ & NS & -0.11 & NS \\
\hline \multicolumn{6}{|l|}{ Verbal fluency } \\
\hline Letter $p$ & $14.1(0.9)$ & $15.8(0.8)$ & NS & 0.02 & NS \\
\hline Letter $f$ & $11.5(5-20)$ & $12.5(3-18)$ & NS & -0.02 & NS \\
\hline Letter I & $10.3(0.66)$ & $10(0.57)$ & NS & 0.25 & NS \\
\hline
\end{tabular}



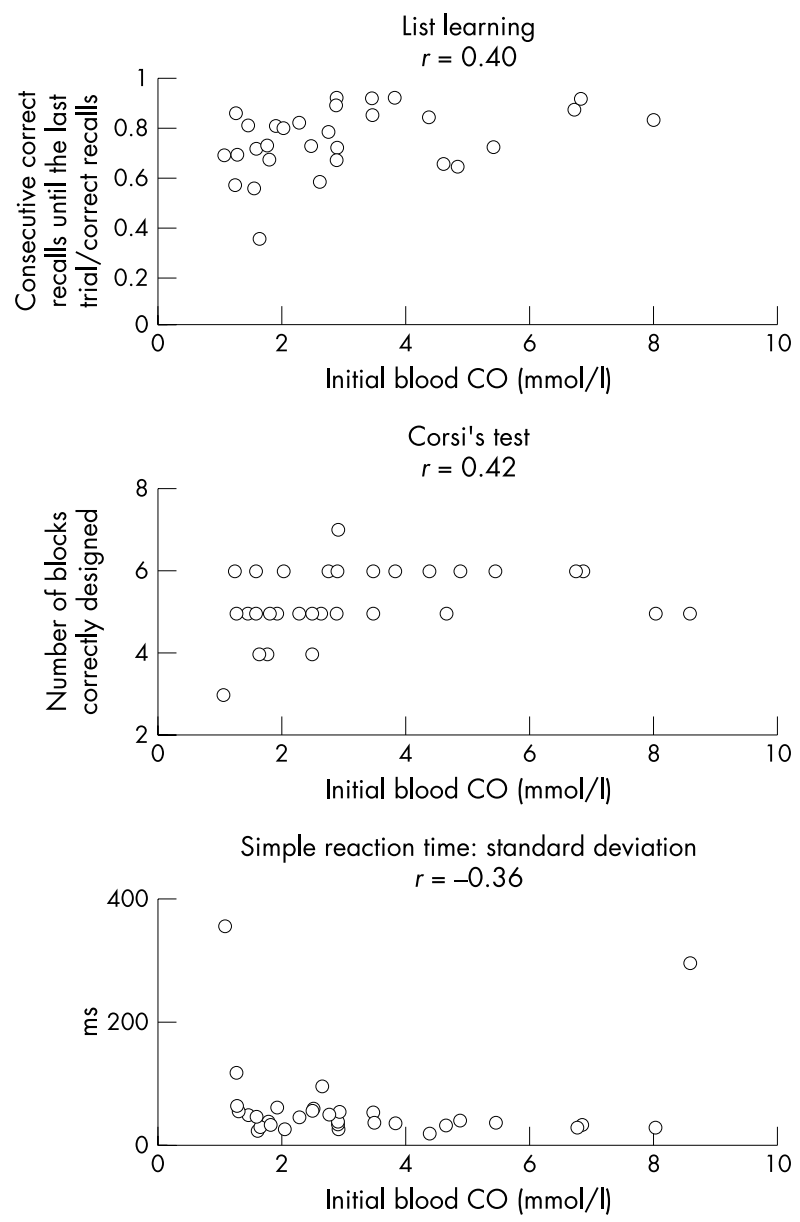

Figure 1 Correlation between initial blood $\mathrm{CO}$ concentration lat the end of exposure, calculated from $\mathrm{CO}$ concentration in the first blood sample) and test results.

influenced the results ${ }^{24}$; however, the administration of the tests was standardised; furthermore, as our hypothesis was that CO poisoned patients would perform memory tests worse than controls, an influence of the examiner would probably have led to the opposite result. Therefore, we do not think that the behaviour of the examiner could explain the results. Fourth, the size of the sample could have been too small to detect a memory impairment in poisoned patients in comparison with controls. However, significant opposite results were observed. Fifth, intoxications could have been too moderate to show an effect of CO on memory. In order to prevent this risk, patients were included only if blood CO concentration in the first sample was at least equal to $1.0 \mathrm{mmol} / \mathrm{l}$ (11\% carboxyhaemoglobin). Sixth, one can theoretically not exclude an effect of oxygen therapy. However, such a delayed effect is not expected. ${ }^{25}$ Finally, it should be noted that oxygen therapy, normobaric with or without hyperbaric, was administered according to standard criteria ${ }^{11}$ and that the aim of the study was not to compare normobaric versus hyperbaric oxygen therapy.

In our patients, who did not complain of neurological symptoms, no radioimaging examination such as magnetic resonance radioimaging (MRI) or single photon emission computed tomography (SPECT) was performed. However, in patients with brain injury following CO poisoning, neuropsychological examination is more sensitive than brain imaging. ${ }^{26}$

Our study focused on a very small subset of patients (according to highly selected population criteria) treated in standardised way ${ }^{11}$ and compared to healthy paired controls.
The results lead us to speculate that the psychological trauma of the intoxication led to the desire for a better performance, resulting in a better effort in the tests and thus in a participative effect. This could explain the better attention in patients compared to controls, which could in turn explain both better performances in several subtypes of memory and a greater fatigability.

In a report of four cases, Weaver et al failed to find abnormal results at extensive neuropsychological memory testing six months after severe CO poisoning in four patients who did not undergo hyperbaric oxygen therapy. ${ }^{27}$

The study methodology could explain why our results, as do those of Weaver et al, differ from most data in the literature, ${ }^{5}$ in which the incidence of sequelae after $\mathrm{CO}$ poisoning may reach $67 \%$. First, our study is a prospective study of CO poisoned patients, without obvious neurological sequelae. In retrospective studies of patients referred for neurological investigations, as the patients suffered from clinical symptoms, they may have had different results. ${ }^{26}$ Second, possible bias or confounding factors related to patients' medical and psychiatric histories were not taken into account in several studies; on the contrary, we did not include patients with such factors, which limited the size of the sample but increased the validity. Indeed, only $16 \%$ of the patients with CO blood level over $1.0 \mathrm{mmol} / \mathrm{l}$ were included: in our experience, many patients suffering from a CO intoxication have one or several factors (psychiatric disease, alcoholism, psychotropic drug treatment, etc) that may lead to neuropsychological disturbances. However, patients who already suffer from such disorders for another reason, or in whom memory function is physiologically lower, because of age or low educational level, may be more susceptible to a possible action of $\mathrm{CO}$ on memory: we did not test this hypothesis. Third, our study was performed on detailed memory fonction assessed with psychometric testing precisely described and statistically analysed. In one of the two studies utilising psychometric testing one month after poisoning, results of subjects treated with normobaric oxygen therapy were worse than those of healthy controls for a subtest of temporospatial orientation, but memory was not extensively studied. ${ }^{28}$ In the other study with psychometric testing one month after poisoning, patients treated with hyperbaric oxygen therapy showed no difference with those treated with normobaric oxygen therapy, but detailed results were not provided and no comparison to a non-poisoned control group was performed. ${ }^{29}$ The other previous published studies were most often conducted without psychometric testing, or the results of the tests were not provided. It is possible that, in these previously published studies, the frequent minor subjective neuropsychological sequelae which were most often reported, resulted not only from potential confounding factors, but also from psychological trauma which may be severe and is related to the occurrence of the intoxication itself, and not to a specific toxic action of CO. The sensation of impending death, followed by a disruption of everyday life after a fire in the home, may lead to anxiety and sleep disturbances, and sometimes may induce or reveal a depressive syndrome. These conditions can lead to vague and various complaints, among them sensation of memory decrease, which is in fact not related to a true impairment of memory function, as evaluated with psychometric tests. The aim of our study was not to answer this question, and patients were tested not for their subjective evaluation of memory loss, but for their objective functioning of memory.

In conclusion, in a small subset of patients highly selected and devoid of any risk factor regarding memory impairment, the objective study of memory one month after intoxication showed that memory is no worse in CO poisoned patients treated with standardised oxygen therapy than in paired controls. We hypothesised that a better effort at attention of these patients, especially when intoxication had been severe, could 
explain better results in several subtests. Further studies should be performed to confirm this result and investigate explanatory hypotheses.

\section{ACKNOWLEDGEMENTS}

We thank Mr Leclercq who provided us with the reaction time and S Borron for critical comments.

\section{Authors' affiliations}

D Deschamps, C Géraud, S Dally, Department of Internal Medicine and Clinical Toxicology, Fernand Widal-Lariboisière Hospital, 75475 Paris Cedex 10, France

F J Baud, Department of Medical and Toxicological Intensive Care, Fernand Widal-Lariboisière Hospital; University Paris 7-Institut National de la Santé et de la Recherche Médicale (INSERM U26) 75475 Paris Cedex 10, France

H Julien, Emergency Medical Services, Paris Fire Department, 55

boulevard de Port Royal, 75013 Paris, France

\section{REFERENCES}

1 Cobb N, Etzel RA. Unintentional carbon monoxide-related deaths in the United States, 1979 through 1988. JAMA 1991;266:659-63.

2 Ernst A, Zibrak JD. Carbon monoxide poisoning. N Engl J Med 1998:339:1603-8.

3 Piantadosi CA, Zhang J, Levin ED, et al. Apoptosis and delayed neuronal damage after carbon monoxide poisoning in the rat. Exp Neurol 1997; 147:103-14.

4 Nabeshima T, Katoh A, Ishimaru $\mathrm{H}$, et al. Carbon monoxide-induced delayed amnesia, delayed neuronal death and change in acetylcholine concentration in mice. J Pharmacol Exp Ther 1991;256:378-84.

5 Gorman DF, Clayton D, Gilligan JE, et al. A longitudinal study of 100 consecutive admissions for carbon monoxide poisoning to the Royal Adelaide Hospital. Anaesth Intens Care 1992;20:311-16.

6 Roche L, Bertoye A, Vincent P. A comparison of two groups of twenty carbon monoxide poisonings treated with normobaric and hyperbaric oxygen. Lyon Méd 1968;49:1483-99.

7 Mathieu D, Nolf M, Durocher A, et al. Acute carbon monoxide poisoning risk of late sequelae and treatment by hyperbaric oxygen. Clin Toxicol 1985;23:315-24

8 Raphaël JC, Elkharrat D, Jars-Guincestre MC, et al. Trial of normobaric and hyperbaric oxygen for acute carbon monoxide intoxication. Lancet 1989;2:414-19.

9 Myers RAM, Snyder SK, Emhoff TA. Subacute sequelae of carbon monoxide poisoning. Ann Emerg Med 1985;14:1163-7.

10 Smith JS, Brandon S. Morbidity from acute carbon monoxide poisoning at three-year follow-up. BN 1973;i:318-21.
11 Tomaszewski C. Carbon monoxide. In: Goldfrank LR, Flomenbaum NE, Lewin NA, et al, eds. Toxicologic emergencies, 6th edn. Stamford, USA: Appleton and Lange, 1998:1551-66.

12 Moureu H, Chavin P, Truffert L, et al. Nouvelle microméthode pour la détermination rapide et précise de l'oxycarbonémie par absorption sélective dans l'infrarouge. Arch Mal Prof 1957;18:116-24.

13 Levasseur L, Galliot-Guilley M, Richter F, et al. Effects of mode of inhalation of carbon monoxide and of normobaric oxygen administration on carbon monoxide elimination from the blood. Hum Exp Toxicol 1996;15:898-903.

14 Choi S. Delayed neurologic sequelae in carbon monoxide intoxication. Arch Neurol 1983;40:433-5.

15 Buschke H. Selective reminding for analysis of memory and learning. Journal of Verbal Learning and Verbal Behavior 1973:12:543-50.

16 Wechsler DA. A standardized memory scale for clinical use. J Psychol 1945;19:87-95.

17 Orsini A, Grossi D, Capitani E, et al. Verbal and spatial immediate memory span: normative data from 1355 adults and 1112 children. Ital J Neurol Sci 1987:8:539-48

18 Leclercq $M$, Chipp PE, Blandel A, et al. A simple reaction times set. European symposium on microcomputers in neuropsychological assessment and rehabilitation, Strasbourg, 1988.

19 Jensen AR, Rohwer WD. The Stroop color-word test: a review. Acta Psychol 1966;25:36-93

20 Borkowski JG, Benton AL, Spreen O. Word fluency and brain damage. Neuropsychologica 1967;5:135-40.

21 Hintze JL. Number Cruncher Statistical System, 2nd edn. Kay Srille, 1987.

22 Amitai Y, Zlotogorski Z, Golan-Katzav V, et al. Neuropsychological impairment from acute low-level exposure to carbon monoxide. Arch Neurol 1998;55:845

23 McNulty J, Maher B, Chu M, et al. Relationship of short-term verbal memory to the need for hyperbaric oxygen treatment after carbon monoxide poisoning. Neuropsychol Behav Neurol 1997:10:174-9.

24 Benignus VA. Importance of experimenter-blind procedure in neurotoxicology. Neurotoxicol Teratol 1993;15:45-9.

25 Scholey AB, Moss MC, Wesnes K. Oxygen and cognitive performance: the temporal relationship between hyperoxia and enhanced memory. Psychopharmacology 1998;140:123-6.

26 Gale S, Hopkins R, Weaver L, et al. MRI, quantitative MRI, SPECT, and neuropsychological findings following carbon monoxide poisoning. Brain Injury 1999;13:229-43

27 Weaver LK, Hopkins RO, Larson-Lohr V. Neuropsychologic and functional recovery from severe carbon monoxide poisoning without hyperbaric oxygen therapy. Ann Emerg Med 1996;27:736-40.

28 Thom SR, Taber RL, Mendiguren II, et al. Delayed neuropsychologic sequelae after carbon monoxide poisoning : prevention by treatment with hyperbaric oxygen. Ann Emerg Med 1995;25:474-80.

29 Scheinkestel CD, Bailey M, Myles P, et al. Hyperbaric or normobaric oxygen for acute carbon monoxide poisoning: a randomized controlled clinical trial. Med J Aust 1999;170:203-10 